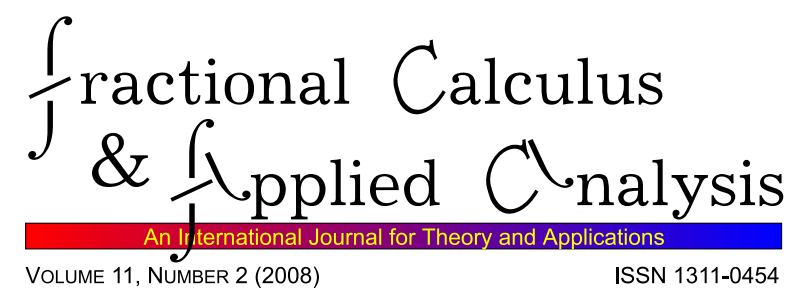

\title{
ON THE GENERALIZED ASSOCIATED LEGENDRE FUNCTIONS
}

\author{
Nina A. Virchenko ${ }^{1}$, Olena V. Rumiantseva ${ }^{2}$ \\ Dedicated to Professor Shyam L. Kalla \\ on the occasion of his 70th birthday
}

\begin{abstract}
This paper is devoted to an important case of Wright's hypergeometric function ${ }_{2} F_{1}^{\tau, \beta}(a, b ; c ; z)={ }_{2} F_{1}^{\tau, \beta}(z)$, to studying its basic properties and to application of ${ }_{2} F_{1}^{\tau, \beta}(z)$ to the generalization of the associated Legendre functions.

Mathematics Subject Classification: 33C60, 33C20, 44A15

Key Words and Phrases: Wright's hypergeometric function, generalized Legendre functions

\section{Introduction}

It is known that the gamma function appeared in the definition of the fractional order differentiation given by L. Euler and later developed by J. Liouville. A detailed discussion of this can be found in [14], [1]. The $\Gamma-$ and $B$-functions are also in the base of many special functions [3], [15]. The diversity of the problems generating special functions has led to a quick increasing in the number of functions used in applications, from the simplest transcendental functions to the hypergeometric functions of different forms, see [8], [9].

One of the most important special functions is the Gauss hypergeometric function ${ }_{2} F_{1}(a, b ; c ; z)$. It should be noted that many algebraic or transcendental functions that occur in the problems of applied mathematics can be
\end{abstract}


expressed in terms of the hypergeometric functions. The Legendre, Bessel, Whittaker and other special functions, and the classical orthogonal polynomials are particular cases of the hypergeometric functions or their various combinations. Let us note that in a systematic study of the generalized probability density, in solving problems of the theory of special functions, differential and integral equations, integral transforms etc., these functions and their applications have played a significant role, see for example [2], [4], [7], [5], [9], [13], [8].

The Legendre functions are one of the most well known particular cases of the hypergeometric function. They have been discovered by Laplace and by Legendre as early as in the $18^{\text {th }}$ century. Later, their importance has grown substantially due to their connections with many problems of mathematical physics, in the potential theory for spheroidal, toroidal and other coordinates [6]. A general theory of the Legendre functions has been constructed by Heine, Hobson, Burns, Bateman, Erdélyi, Watson and etc., as $[5],[6],[8],[12]$.

There exist various generalizations of the Legendre and the associated Legendre functions $P_{\nu}^{\mu}(z), Q_{\nu}^{\mu}(z)$. In 1957 Kuipers and Meulenbeld [11] introduced into consideration the generalized associated Legendre functions of the first and the second kind $P_{k}^{m, n}(z)$ and $Q_{k}^{m, n}(z)$ respectively, see [11], [16], [19].

In this paper we consider the generalized (in the sense of Wright [20], [8]) hypergeometric Gauss function and its applications to the generalization of the Legendre functions ${ }^{\tau} P_{\nu}^{\mu}(z),{ }^{\tau, \beta} P_{k}^{m, n}(z),{ }^{\tau, \beta} Q_{k}^{m, n}(z)$. These functions are useful in such areas of applications as mathematical physics, heat conduction, astronomy, quantum mechanics, approximation theory, probability theory etc., [1], [7], [17], [18], [19].

\section{2. $(\tau, \beta)$-generalized Gauss hypergeometric function}

Let us introduce the $(\tau, \beta)$-generalized Gauss hypergeometric function of the following form:

$$
\begin{aligned}
{ }_{2} F_{1}^{\tau, \beta}(a, b ; c ; z) \equiv & { }_{2} F_{1}^{\tau, \beta}(z)=\frac{\Gamma(c)}{\Gamma(a) \Gamma(b) \Gamma(c-b)} \int_{0}^{1} t^{b-1}(1-t)^{c-b-1} \\
& \left.\times{ }_{2} \Psi_{1}\left[\begin{array}{c}
(a, 1),(c, \tau) ; \\
(c, \beta) ;
\end{array}\right] z t^{\tau}\right] d t
\end{aligned}
$$


where $a, b, c$ can be complex, $\{\tau, \beta\} \subset R, \tau>0, \beta>0, \operatorname{Rec}>\operatorname{Reb}>$ 0, Rea $>0, \tau-\beta \leq 1, \Gamma(\ldots)$ is the classical gamma function [5], ${ }_{2} \Psi_{1}$ is the Fox-Wright function [20]. When $\beta=\tau=1$, (1) gives the classical hypergeometric function ${ }_{2} F_{1}(a, b ; c ; z)$, see [5].

TheOREM 2.1. (Integral representations of ${ }_{2} F_{1}^{\tau, \beta}(z)$ ) For the function ${ }_{2} F_{1}^{\tau, \beta}(a, b ; c ; z)$ the following integral representations are valid:

$$
\begin{gathered}
{ }_{2} F_{1}^{\tau, \beta}(a, b ; c ; z)=A \int_{0}^{\infty} t^{b-1}(1+t)^{-c}{ }_{2} \Psi_{1}\left[\begin{array}{c}
(a, 1),(c, \tau) ; \\
(c, \beta) ;
\end{array} \mid z\left(\frac{t}{1+t}\right)^{\tau}\right] d t ;(2) \\
{ }_{2} F_{1}^{\tau, \beta}(a, b ; c ; z)=2 A \int_{0}^{\infty}(s h t)^{2 b-1}(c h t)^{1-2 c}{ }_{2} \Psi_{1}\left[\begin{array}{c}
(a, 1),(c, \tau) ; \\
(c, \beta) ;
\end{array} \mid z(t h t)^{2 \tau}\right] d t
\end{gathered}
$$

where

$$
\begin{gathered}
A=\frac{\Gamma(c)}{\Gamma(a) \Gamma(b) \Gamma(c-b)} ; \\
{ }_{2} F_{1}^{\tau, \beta}\left(a, b ; c ; \frac{1}{x^{\beta}}\right)=x^{c-\beta} \frac{\Gamma(c)}{\Gamma(c-\beta) \Gamma(\beta)} \int_{x}^{\infty} \frac{(t-x)^{\beta-1}}{t^{c}}{ }_{2} F_{1}^{\tau, \beta}\left(a, b ; c-\beta ; \frac{1}{t^{\beta}}\right) d t
\end{gathered}
$$

(the generalized Koornwinder' formula [10]),

$\operatorname{Re}(c-\beta)>0, \operatorname{Rea}>0, \operatorname{Re} b>0,|x|>1$.

The validity of relations (2), (3), (5) is proved with the help of the series representations of ${ }_{2} F_{1}^{\tau, \beta}(z)$, applications of the properties of the $\Gamma-$ and $B$-functions, the legality of interchanging of the order of integration and summation.

For example, let us prove (5):

$$
\begin{gathered}
\int_{x}^{\infty} \frac{(t-x)^{\beta-1}}{t^{c}}{ }_{2} F_{1}^{\tau, \beta}\left(a, b ; c-\beta ; \frac{1}{t^{\beta}}\right) d t \\
=\frac{\Gamma(c-\beta)}{\Gamma(a) \Gamma(b)} \sum_{n=0}^{\infty} \frac{\Gamma(a+n) \Gamma(b+\tau n)}{\Gamma(c-\beta+\beta n)} \frac{1}{n !} \int_{x}^{\infty}(t-x)^{\beta-1} t^{-c-\beta n} d t \\
=\frac{\Gamma(c-\beta)}{\Gamma(a) \Gamma(b)} \sum_{n=0}^{\infty} \frac{\Gamma(a+n) \Gamma(b+\tau n)}{\Gamma(c-\beta+\beta n)} \frac{1}{n !} x^{-c-\beta n+\beta} B(\beta, c-\beta+\beta n) \\
=x^{\beta-c} \frac{\Gamma(c-\beta) \Gamma(\beta)}{\Gamma(c)}{ }_{2} F_{1}\left(a, b ; c-\beta ; \frac{1}{x^{\beta}}\right) .
\end{gathered}
$$


Theorem 2.2. (Addition formula) For the $(\tau, \beta)$-generalized Gauss hypergeometric function ${ }_{2} F_{1}^{\tau, \beta}(z)$ the following addition formula is valid:

$$
\begin{aligned}
{ }_{2} F_{1}^{\tau, \beta}(a, b ; c ; x+y) & =\frac{\Gamma(c)}{\Gamma(a) \Gamma(b)} \sum_{n=0}^{\infty} \frac{\Gamma(a+n) \Gamma(b+\tau n)}{\Gamma(c+n \beta)} \frac{y^{n}}{n !} \\
& \times{ }_{2} F_{1}^{\tau, \beta}(a+n, b+n \tau ; c+n \beta ; x) .
\end{aligned}
$$

$\mathrm{P}$ r o o f. It follows from the differentiation formula for ${ }_{2} F_{1}^{\tau, \beta}(z)$ :

$$
\begin{aligned}
\frac{d^{n}}{d z^{n}}{ }_{2} F_{1}^{\tau, \beta}(a, b ; c ; z) & =\frac{\Gamma(a+n) \Gamma(c) \Gamma(b+\tau n)}{\Gamma(a) \Gamma(b) \Gamma(c+\beta n)} \\
& \times{ }_{2} F_{1}^{\tau, \beta}(a+n, b+\tau n ; c+\beta n ; z),
\end{aligned}
$$

and Taylor' theorem [15]: if $f(x)$ is analytical function, its series converges as $|x|<\rho$, then for $|y|<\rho$ we have

$$
f(x+y)=\sum_{n=0}^{\infty} f^{(n)}(x) \frac{y^{n}}{n !} .
$$

Theorem 2.3. (Product formula) For the $(\tau, \beta)$-generalized Gauss hypergeometric function ${ }_{2} F_{1}^{\tau, \beta}(z)$ the following formula is valid:

$$
\begin{aligned}
{ }_{2} F_{1}^{\tau, \beta}(a, b ; c ; x y) & =\frac{\Gamma(c)}{\Gamma(a) \Gamma(b)} \sum_{n=0}^{\infty} \frac{\Gamma(a+n) \Gamma(b+\tau n)}{\Gamma(c+n \beta)} \frac{x^{n}(y-1)^{n}}{n !} \\
& \times{ }_{2} F_{1}^{\tau, \beta}(a+n, b+\tau n ; c+\beta n ; x) .
\end{aligned}
$$

Here we use (7) and the Taylor theorem [15]:

$$
f(x y)=\sum_{n=0}^{\infty} \frac{(y-n)^{n}}{n !} x^{n} f^{(n)}(x) .
$$

Theorem 2.4. (Generalization of the Erdélyi theorem [5]) If Rec $>$ $\operatorname{Re} \lambda>0, z \neq 1,|\arg (1-z)|<\pi, \operatorname{Rec}>\operatorname{Re} b>0, \operatorname{Re} 2 b>\operatorname{Rea}>0, \tau \in$ $R, \tau>0$, then the following formula is valid:

$$
\begin{aligned}
{ }_{2} F_{1}^{\tau}(a, b ; c ; z) & =\frac{\Gamma(c) \Gamma^{-1}(\lambda-\beta+1)}{\Gamma(c-b) \Gamma(2 b-\lambda)} \int_{0}^{1} x^{b-1}{ }_{2} F_{1}(b-c+1,1 ; \lambda-b+1 ; x) \\
& \times{ }_{2} F_{1}^{\tau}\left(a, b ; 2 b-\lambda ; z x^{\tau}\right) d x
\end{aligned}
$$


P r o o f. Let $\beta=\tau$. From (1) we have

$$
{ }_{2} F_{1}^{\tau}(a, b ; c ; z)=\frac{\Gamma(c)}{\Gamma(b) \Gamma(c-b)} \int_{0}^{1}(1-x)^{c-b-1} x^{b-1}\left(1-z x^{\tau}\right)^{-a} d x .
$$

Let us consider the expression $x^{b-1}\left(1-z x^{\tau}\right)^{-a}$. Taking into account the following formula $([5])$

$$
\left(1-z x^{\tau}\right)^{-a}=\frac{1}{\Gamma(a)} \sum_{n=0}^{\infty} \Gamma(a+n) \frac{z^{n}}{n !} x^{\tau n}, \quad\left(\left|z x^{\tau}\right|<1\right),
$$

we obtain:

$$
\begin{gathered}
x^{b-1}\left(1-z x^{\tau}\right)=x^{b-1} \sum_{n=0}^{\infty} \frac{(a)_{n}}{n !} x^{\tau n} z^{n} \\
\sum_{n=0}^{\infty} \frac{(a)_{n}}{n !} x^{b+\tau n-1} z^{n}=\frac{\Gamma(b)}{\Gamma(2 b-\lambda)} \frac{d^{b-\lambda}}{d x^{b-\lambda}}\left[\sum_{n=0}^{\infty} \frac{(a)_{n}}{n !} \frac{\Gamma(b+\tau n)}{\Gamma(2 b-\lambda+\tau n)} \frac{\Gamma(2 b-\lambda)}{\Gamma(b)}\right. \\
\left.\times z^{n} x^{2 b+\tau n-1-\lambda}\right]=\frac{\Gamma(b)}{\Gamma(2 b-\lambda)} \frac{d^{b-\lambda}}{d x^{b-\lambda}}\left[{ }_{2} F_{1}^{\tau}\left(a, b ; 2 b-\lambda ; z x^{\tau}\right) x^{2 b-\lambda-1}\right] .
\end{gathered}
$$

Using the derivative

$$
\frac{d^{b-\lambda} x^{2 b+\tau n-\lambda-1}}{d x^{b-\lambda}}=\frac{\Gamma(2 b-\lambda+\tau n)}{\Gamma(b+\tau n)} x^{b+\tau n-1}
$$

and the fractional integration by parts we get:

$$
\begin{gathered}
{ }_{2} F_{1}^{\tau}(a, b ; c ; z)=\frac{\Gamma(c)}{\Gamma(c-b) \Gamma(2 b-\lambda)} \\
\times \int_{0}^{1}(1-x)^{c-b-1} \frac{d^{b-\lambda}}{d x^{b-\lambda}}\left\{x^{2 b-\lambda-1}{ }_{2} F_{1}^{\tau}\left(a, b ; 2 b-\lambda ; z x^{\tau}\right)\right\} d x \\
=\frac{\Gamma(c)}{\Gamma(c-b) \Gamma(2 b-\lambda)} \int_{0}^{1} x_{2}^{2 b-\lambda-1}{ }_{2} F_{1}^{\tau}\left(a, b ; 2 b-\lambda ; z x^{\tau}\right) \frac{d^{b-\lambda}}{d x^{b-\lambda}}\left\{(1-x)^{c-b-1}\right\} \\
=\frac{\Gamma(c)}{\Gamma(c-b) \Gamma(2 b-\lambda)} \int_{0}^{1} x^{2 b-\lambda-1}{ }_{2} F_{1}^{\tau}\left(a, b ; 2 b-\lambda ; z x^{\tau}\right) \sum_{n=0}^{\infty} \frac{(b-c+1)_{n}}{n !}
\end{gathered}
$$




$$
\begin{gathered}
\times \frac{x^{n+\lambda-b} \Gamma(n+1)}{\Gamma(\lambda-b+1+n)} \frac{\Gamma(\lambda-b+1)}{\Gamma(\lambda-b+1)}=\frac{\Gamma(c) \Gamma^{-1}(\lambda-\beta+1)}{\Gamma(c-b) \Gamma(2 b-\lambda)} \\
\times \int_{0}^{1} x^{b-1}{ }_{2} F_{1}(b-c+1,1 ; \lambda-b+1 ; x){ }_{2} F_{1}^{\tau}\left(a, b ; 2 b-\lambda ; z x^{\tau}\right) d x .
\end{gathered}
$$

Thus the theorem is proved.

\section{The generalized associated Legendre functions}

For the first time the functions $P_{k}^{m, n}(z), Q_{k}^{m, n}(z)$ were introduced as linearly independent solutions of the following generalized Legendre differential equation [11]:

$$
\left(1-z^{2}\right) \frac{d^{2} u}{d z^{2}}-2 z \frac{d u}{d z}+\left[k(k+1)-\frac{m^{2}}{2(1-z)}-\frac{n^{2}}{2(1+z)}\right] u=0 .
$$

Let us note that as $m=n=\mu, k=\nu$, we have the known Legendre functions $P_{\nu}^{\mu}(z), Q_{\nu}^{\mu}(z)$, [6]. If $z \in C$ and $k, m, n$ are the real parameters, then the solutions of the differential equation (14) may be written in the form:

$$
u=P\left(\begin{array}{cccc}
1 & \infty & -1 & \\
\frac{m}{2} & -k & \frac{n}{2} & z \\
-\frac{m}{2} & k+1 & -\frac{n}{2} &
\end{array}\right),
$$

where $\arg z, \arg (z \pm 1)$ have their principal values. Evidently, the generalized functions $P_{k}^{m, n}(z), Q_{k}^{m, n}(z)$ are of the class of the hypergeometric functions. The functions $P_{k}^{m, n}(z)$ and $Q_{k}^{m, n}(z)$ are defined for all points of the complex $z$-plane cut along the real axis from $-\infty$ to 1 .

Let us introduce the $(\tau, \beta)$-generalized associated Legendre functions ${ }^{\tau, \beta} P_{k}^{m, n}(z),{ }^{\tau, \beta} Q_{k}^{m, n}(z)$ of the following form:

$$
\begin{gathered}
\tau, \beta P_{k}^{m, n}(z)=\frac{1}{\Gamma(1-m)} \frac{(z+1)^{\frac{n}{2}}}{(z-1)^{\frac{m}{2}}} \\
\times{ }_{2} F_{1}^{\tau, \beta}\left(k-\frac{m-n}{2}+1,-k-\frac{m-n}{2} ; 1-m ; \frac{1-z}{2}\right),
\end{gathered}
$$

where$$
|1-z|<2 ; k+\frac{m+n}{2} \neq-1,-2, \ldots ; k-\frac{m-n}{2} \neq 0, \pm 1, \pm 2, \ldots ;
$$$$
m \neq 1,2, \ldots ;|\arg (z \pm 1)|<\pi,\{\tau, \beta\} \subset R, \tau>0, \tau-\beta \leq 1 ;
$$ 


$$
\begin{array}{r}
\tau, \beta \\
Q_{k}^{m, n}(z)=e^{m \pi i} \frac{2^{k-\frac{(m-n)}{2}} \Gamma\left(k+\frac{(m+n)}{2}+1\right) \Gamma\left(k+\frac{(m-n)}{2}+1\right)}{(z-1)^{k+\frac{n}{2}+1}(z+1)^{-\frac{n}{2}} \Gamma(2 k+2)} \\
\quad \times{ }_{2} F_{1}^{\tau, \beta}\left(k-\frac{m-n}{2}+1, k+\frac{m+n}{2}+1 ; 2 k+2 ; \frac{2}{1-z}\right),
\end{array}
$$

with

$$
\begin{gathered}
k+\frac{m+n}{2} \neq-1,-2, \ldots, k \pm \frac{m-n}{2} \neq 0, \pm 1, \pm 2, \ldots ; 2 k \neq-2,-3, \ldots \\
|z-1|>2,|\arg (z \pm 1)|<\pi,\{\tau, \beta\} \subset R, \tau>0, \tau-\beta \leq 1
\end{gathered}
$$

In the sequel we discuss some properties of the functions ${ }^{\tau, \beta} P_{k}^{m, n}(z)$, ${ }^{\tau, \beta} Q_{k}^{m, n}(z)$.

THEOREM 3.1. If the conditions (16) are valid, then the following integral representations for the function ${ }^{\tau, \beta} P_{k}^{m, n}(z)$ hold:

$$
\begin{aligned}
& { }^{\tau, \beta} P_{k}^{m, n}(z)=\frac{(z+1)^{\frac{n}{2}}(z-1)^{-\frac{m}{2}}}{\Gamma\left(k+1-\frac{m-n}{2}\right) \Gamma\left(-k-\frac{m-n}{2}\right) \Gamma\left(k+1-\frac{m+n}{2}\right)} \\
& \times \int_{0}^{1} t^{-k-\frac{m-n}{2}-1}(1-t)^{k-\frac{m+n}{2}} \\
& \times{ }_{2} \Psi_{1}\left[\begin{array}{l|l}
\left(k-\frac{m-n}{2}+1,1\right),(1-m, \tau) ; & \frac{1-z}{2} \cdot t^{\tau} \\
(1-m, \beta) ;
\end{array}\right] d t,
\end{aligned}
$$

where ${ }_{2} \Psi_{1}$ is the Fox-Wright function [20];

$$
\begin{aligned}
& { }^{\tau, \beta} P_{k}^{m, n}(\operatorname{ch} \alpha)=K \operatorname{ch}^{n} \frac{\alpha}{2} \cdot s h^{-m} \frac{\alpha}{2} \cdot \int_{0}^{\infty} e^{\left(k+\frac{m-n}{2}\right) t}\left(1-e^{-t}\right)^{k-\frac{m+n}{2}} \\
& \times{ }_{2} \Psi_{1}\left[\begin{array}{c}
\left(k-\frac{m-n}{2}+1,1\right),(1-m, \tau) ; \mid \\
(1-m, \beta) ;
\end{array} \operatorname{sh}^{2} \frac{\alpha}{2} \cdot e^{-\tau t}\right] d t ; \quad(20) \\
& \tau, \beta P_{k}^{m, n}(\cos \omega)=K 2 \cdot \cos ^{n} \frac{\omega}{2} \cdot\left(-\sin \frac{\omega}{2}\right)^{-m} \int_{0}^{\frac{\pi}{2}}(\cos t)^{-2 k-m+n-1}(\sin t)^{2 k-m-n+1} \\
& \times{ }_{2} \Psi_{1}\left[\begin{array}{c}
\left(k-\frac{m-n}{2}+1,1\right),(1-m, \tau) ; \\
(1-m, \beta) ;
\end{array} \sin ^{2} \frac{\omega}{2} \cdot \cos ^{2 \tau} t\right] d t
\end{aligned}
$$




$$
\begin{aligned}
& { }^{\tau, \beta} P_{k}^{m, n}(\operatorname{ch} \alpha)=K \cdot 2 \operatorname{ch}^{n} \frac{\alpha}{2} \cdot \operatorname{sh}^{-m} \frac{\alpha}{2} \int_{9}^{\infty}(\operatorname{ch} \omega)^{2 m-1} \cdot(\operatorname{sh} \omega)^{2 k-m-n+1} \\
& \times_{2} \Psi_{1}\left[\begin{array}{c}
\left(k-\frac{m-n}{2}+1,1\right),(1-m, \tau) ; \\
(1-m, \beta) ;
\end{array} \mid-\operatorname{sh}^{2} \frac{\alpha}{2} \cdot(\operatorname{ch} \omega)^{-2 \tau}\right] d \omega ;
\end{aligned}
$$

with

$$
K=\frac{2^{\frac{n-m}{2}} \Gamma^{-1}\left(k-\frac{m+n}{2}+1\right)}{\Gamma\left(k-\frac{m-n}{2}+1\right) \Gamma\left(-k-\frac{m-n}{2}\right)} .
$$

Theorem 3.2. If the conditions (18) are valid, then some integral representations for the function ${ }^{\tau, \beta} Q_{k}^{m, n}(z)$ have the following form:

$$
\begin{aligned}
& { }^{\tau, \beta} Q_{k}^{m, n}(z)=e^{\pi i m} \frac{2^{k-\frac{m-n}{2}}(z-1)^{-k-\frac{n}{2}-1}(z+1)^{\frac{n}{2}} \Gamma\left(k+\frac{m+n}{2}\right) \Gamma\left(k+\frac{m-n}{2}+1\right)}{\Gamma\left(k-\frac{m-n}{2}+1\right) \Gamma\left(k+\frac{m+n}{2}+1\right) \Gamma\left(k-\frac{m+n}{2}+1\right)} \\
& \times \int_{0}^{1} t^{k+\frac{m+n}{2}}(1-t)^{k-\frac{m+n}{2}}{ }_{2} \Psi_{1}\left[\begin{array}{l|l}
\left(k-\frac{m-n}{2}+1,1\right),(2 k+2, \tau) ; \mid & \frac{2}{1-z} t^{\tau} \\
(2 k+2, \beta) ;
\end{array}\right] d t ; \\
& { }_{\tau, \beta} Q_{k}^{m, n}(\operatorname{ch} \alpha)=M\left(\operatorname{sh} \frac{\alpha}{2}\right)^{-2 k-n-2}\left(\operatorname{ch} \frac{\alpha}{2}\right)^{n} \int_{0}^{\infty}(\operatorname{cht})^{-4 k-3}(\operatorname{sh} t)^{2 k-m-n+1} \\
& \times_{2} \Psi_{1}\left[\begin{array}{l|l}
\left(k-\frac{m-n}{2}+1,1\right),(2 k+2, \tau) ; & -s h^{2} \frac{\alpha}{2} \cdot c h^{-2 \tau} \omega \\
(2 k+2, \beta) ;
\end{array}\right] d \omega,
\end{aligned}
$$

where

$$
M=\frac{e^{i \pi m} 2^{\frac{n-m}{2}} \Gamma\left(k+\frac{m+n}{2}+1\right)}{\Gamma\left(k-\frac{m-n}{2}+1\right) \Gamma\left(k-\frac{m+n}{2}+2\right)} .
$$

The proofs of Theorems 3.1, 3.2 are straightforward with help of the series representation of ${ }_{2} F_{1}^{\tau, \beta}(z)$, the interchanging of the order of summation and integration, and using suitable substitutions.

TheOREm 3.3. (Integral representation of ${ }^{\tau, \beta} P_{k}^{m, n}(z)$ by the hypergeometric functions) If $k+\frac{m+n}{2}$ is a positive integer, and $k-\frac{m-n}{2}$ and $m$ are not integers, $\{\tau, \beta\} \subset R, \tau>0, \beta>0,(\tilde{\beta}-\lambda)$ is positive integer, $|\arg (1-z)|<\pi, z \neq 1$, then the following formula is valid:

$$
{ }^{\tau, \beta} P_{k}^{m, n}(z)=\frac{(z+1)^{\frac{n}{2}}}{(z-1)^{\frac{m}{2}}} \frac{\Gamma^{-1}(\lambda-\tilde{\beta}+1)}{\Gamma\left(1+k-\frac{m+n}{2}\right) \Gamma\left(-k-\frac{m-n}{2}+\tilde{\beta}-\lambda\right)}
$$




$$
\begin{gathered}
\times \int_{0}^{1} t^{-k-\frac{m-n}{2}-1}{ }_{3} \tilde{F}_{2}\left(-k-\frac{m-n}{2}+1,-k-\frac{m-n}{2}, 1-m ;\right. \\
\left.\quad-k-\frac{m-n}{2}+\tilde{\beta}-\lambda, 1-m ; \frac{1-z}{2} \cdot t^{\tau}\right) \\
\times{ }_{2} F_{1}\left(-k+\frac{m+n}{2}, 1 ; \lambda+k+\frac{m+n}{2}+1 ; t\right) d t,
\end{gathered}
$$

where ${ }_{3} \tilde{F}_{2}$ is the Wright hypergeometric function ([20]) and ${ }_{2} F_{1}$ is the Gauss hypergeometric function.

The proof is similar to the proof of Theorem 2.4.

COROLlary. Let the conditions of Theorem 3.3 hold. Then the function ${ }^{\tau} P_{k}^{m, n}(z)$ is represented by the expression:

$$
\begin{gathered}
{ }^{\tau} P_{k}^{m, n}(z)=\frac{(z+1)^{\frac{n}{2}}}{(z-1)^{\frac{m}{2}}} \frac{\Gamma^{-1}\left(\lambda+k+\frac{m-n}{2}-1\right)}{\Gamma\left(k-\frac{m+n}{2}+1\right) \Gamma(-2 k-m+n-\lambda)} \int_{0}^{1} t^{-k-\frac{m-n}{2}-1} \\
\quad \times{ }_{2} F_{1}\left(-k+\frac{m+n}{2}, 1 ; \lambda+k+\frac{m-n}{2}+1 ; t\right) \\
\times{ }_{2} F_{1}^{\tau}\left(k-\frac{m-n}{2}+1,-k-\frac{m-n}{2} ;-2 k-m+n-\lambda ; \frac{1-z}{2} t^{\tau}\right) d t .
\end{gathered}
$$

For practical goals the following theorem is useful.

TheOREm 3.4. If $\operatorname{Re} \mu<\frac{1}{2}, \tau \in R, \tau>0, z=$ ch $\alpha$, then for the $\tau$-generalized associated Legendre function ${ }^{\tau} P_{\nu}^{\mu}(z)$ the following formula is valid:

${ }^{\tau} P_{\nu}^{\mu}(\operatorname{ch} \alpha)=N \cdot \int_{-\alpha}^{\alpha} e^{t(\nu+\mu+1)}\left(e^{\alpha}-e^{t}\right)^{-\frac{\mu+\frac{1}{2}}{\tau}-1}\left[1-\left(\left(e^{\alpha}-e^{t}\right) / 2 s h \alpha\right)^{\frac{1}{\tau}}\right]^{-\mu-\frac{1}{2}} d t$

where

$$
N=2^{\mu+\frac{2 \mu-1}{2 \tau}} \tau^{-1} \Gamma^{-1}(1-\mu) \Gamma(1-2 \mu) \Gamma^{-2}\left(\frac{1}{2}-\mu\right)(\operatorname{sh} \alpha)^{-\mu-\frac{1}{2 \tau}+\frac{\mu}{\tau}} .
$$

P r o o f. Taking into account the expression for $P_{\nu}^{\mu}$ with $z \rightarrow \frac{2 \sqrt{z^{2}-1}}{z+\sqrt{z^{2}-1}}$ ([5]), using the substitutions $z=\operatorname{ch} \alpha,(\operatorname{ch} \alpha+\operatorname{sh} \alpha) \cdot 2^{\tau}-2 \operatorname{sh} \alpha(1-\cos t)^{\tau}=$ $e^{r} \cdot 2^{\tau}$, after transformations, we obtain (27). 


\section{References}

[1] S.K. Agarwal, S.L. Kalla, A generalized gamma distribution and its applications in reliability. Comm. Statist. Theory Methods 25 (1996), 201-210.

[2] F. Al-Musallam, S.L. Kalla, Asymptotic expansions for generalized gamma and incomplete gamma functions. Appl. Anal. 66 (1997), 173187.

[3] L.C. Andrews, R. Askey, R. Roy, Special Functions. Cambridge University Press, New York (1999).

[4] K. Aomoto, Hypergeometric functions: The past, today and ... . Sugaku Expositions 9 (1996), 99-116.

[5] A. Erdélyi, W. Magnus, F. Oberhettinger and F.G. Tricomi,Higher Transcendental Functions. Bateman Project, Vols. 1-3. Mc Graw-Hill, New York (1953-1954).

[6] E.W. Hobson, Spherical and Ellipsoidal Harmorics. Cambridge University Press, London (1955).

[7] Shyam L. Kalla, Bader N. Al-Sagabi, Further results on a unified form of gamma-type distributions. Fract. Calculus and Appl. Anal. 4, No 1 (2001), 91-100.

[8] A.A. Kilbas and M. Saigo, H-Transforms. Theory and Applications. Ser. "Analytic Methods and Special Functions", Vol. 9, CRC Press, London and New York (2004).

[9] V.S. Kiryakova, Generalized Fractional Calculus and Applications. Pitman Research Notes in Math., Vol. 301, Longman \& John Wiley and Sons, New York (1994).

[10] T. Koornwinder, A new proof of a Paley-Wiener type theorem for the Jacobi transform. Arkiv f. Math. 13, No 3 (1975), 145-159.

[11] L. Kuipers, B. Meulenbeld, On the generalization of Legendre's associated differential equation. Proc. Konkl. Nederl. Akad. Wet. A. 60, No 4 (1957), 436-450. 
[12] F.G. Mehler. Veber eine mit den Kügel und Cylinder Funktionen verwandte Funktion und ihre Anwendung in der Theorie der Elektricitatsverteilung. Math. Ann. 18, No 1 (1881), 161-194.

[13] S.G. Samko, A.A. Kilbas, O.I. Marichev, Fractional Integrals and Derivatives. Theory and Applications. Gordon and Breach, New York (1993).

[14] Gopala Krishna Srinivasan, The Gamma Function: An Eclectic Tour. The American Mathematical Monthly 114, No 4 (2007), 297-315.

[15] E.C. Titchmarsh, Introduction to the Theory of Fourier Integrals. Oxford Univ. Press, Oxford (1937).

[16] N.A. Virchenko, On some applications of the generalized associated Legendre's function. Ukr. Math. J. 39, No 2 (1987), 149-156.

[17] N. Virchenko, On some generalizations of the functions of hypergeometric type. Fract. Calculus and Appl. Anal. 2, No 3 (1999), 233-244.

[18] N. Virchenko, S.L. Kalla, A. Al-Zamel, Some results on a generalized hypergeometric function. Integral Transforms and Spec. Funct. 12, No 1 (2001), 89-100.

[19] N. Virchenko, I. Fedotova, Generalized Associated Legendre Functions and Their Applications. World Scientific, Singapore - London (2001).

[20] E.M. Wright. The asymptotic expansion of the generalized hypergeometric function. J. London Math. Soc. 10 (1935), 286-293.

1,2 Department of Mathematics and Physics

National Technical University of Ukraine "KPI"

Kyiv, UKRAINE

Received: February 20, 2008

* Corr. address: Polarna Str., No 11, apt. 18

Kyiv - 04201, UKRAINE

e-mail:nvirchenko@hotmail.com 